\title{
- Planificación Óptima de Redes utilizando Algoritmos Evolutivos
}

\author{
* Rosmeri Mayta H.
}

\section{RESUMEN}

El artículo propone la utilización de Algoritmos Evolutivos Multiobjetivos para la planificación de centrales telefónicas a corto, mediano y largo plazo. Los resultados experimentales para una ciudad validan la presente propuesta, por la rapidez, la variedad y la calidad de las soluciones.

Palabras Claves: Planificación de redes. Optimización multiobjetivo. Algoritmos evolutivos.

\section{ABSTRACT}

This article proposes using MultiTargeted Evolutionary Algorithms for Telephone Exchange Planning, on the short, medium and long run. Experimental resuls for a city make the present proposal valuable because of the quick, varied and quality solutions obtained.

Key Words: Net-Planning. Multi-Targeted optimization. Evolutionary algorithms.

\section{INTRODUCCIÓN}

El vertiginoso crecimiento del consumo y variedad de los servicios de telecomunicaciones, genera una necesidad cada vez mayor de implementar herramientas eficientes para la planificación de las redes de telecomunicaciones, a fin de minimizar los altos costos de inversión y mantenimiento. Básicamente, el problema a resolver consiste en calcular la cantidad de centrales necesarias para cubrir la demanda de un área y la correspondiente ubicación eficiente de las mismas, a fin minimizar los costos basados en: los datos de la población, la demanda de tráfico y el costo de la infraestructura requerida para atender la demanda proyectada.

Además, una vez obtenido un conjunto de soluciones óptimas, y habiendo decidido adoptar e implementar una de ellas, se plantea resolver el problema de ubicar nuevas a corto, mediano y largo plazo. Actualmente, existen herramientas de planificación como PLANITU [6] que permiten realizar la planificación de centrales, calculando una ubicación de centrales que atiende a necesidades de telecomunicaciones. Esta herramienta convencional, resuelve el problema en cuestión proponiendo una única solución, calculada mediante métodos tradicionales basados en el álgebra lineal [6]. Este método es adecuado cuando se estudia la posibilidad de instalar una nueva central, pero no es eficiente cuando se esperan ubicar varias centrales, ya que se necesitaría muchísimo tiempo de procesamiento para analizar cada una de las posibles combinaciones, con el agravante de obtener resultados que no garantizan ser una solución óptima. Adicionalmente, herramientas existentes de planificación como PLANITU, tienen la restricción adicional de un costo muy elevado de adquisición y mantenimiento, lo que complica su utilización en instituciones sin suficientes recursos económicos. Históricamente, este tipo de problemas, se ha intentado resolver por medio de programación lineal $[2,3]$, pero esta metodología presenta dificultades en su formulación.

Así mismo, se ha intentado utilizar búsqueda exhaustiva, pero esto sólo es posible para redes muy pequeñas, lo que dificulta su utilización práctica si se considera el tamaño de las actuales redes de telecomunicaciones [7]. Alternativamente, algoritmos "Branch and Bound" eran también utilizados. Sin embargo, debido a la creciente complejidad del diseño de las redes de telecomunicaciones, se han desarrollado también varios algoritmos heurísticos [3] para solucionar grandes instancias del problema de ubicación de centrales. Dos aproximaciones heurísticas conocidos como "ADD" [5] y "DROP"[1] fueron usadas como algoritmos heurísticos para versiones de gran escala del problema en cuestión. Un intento más reciente de encontrar soluciones al referido problema, se basa en las ya conocidas técnicas de inteligencia artificial, conocidas como "Tabu Search". Esta técnica es una aproximación heurística que facilita la derivación de varias alternativas de solución, tales como los algoritmos descendientes. En todos estos casos, la solución encontrada minimiza una única función objetivo, como el costo de inversión para atender una demanda conocida. Sin embargo, no siempre

* Instituto de Investigación

Facultad de Ingeniería Industrial, UNMSM

E-mail: rmaytah@unmsm.edu.pe 
la solución que atiende la demanda actual es la que minimizará los costos en el mediano o largo plazo. En consecuencia, este trabajo propone resolver el problema de planificación de centrales de telecomunicaciones considerando simultáneamente:

- La demanda actual $y_{1}$ (año 2002 para el problema de prueba),

- La demanda a mediano plazo y (año 2004 en el referido problema de prueba),

- La demanda a largo plazo $y_{3}$ (considerando el año 2007 para este trabajo, por falta de estimaciones suficientes para años posteriores).

Posteriormente, se aplicará el mismo concepto de optimización Multiobjetivos para ubicar una nueva central, basado en una solución en particular del conjunto de soluciones obtenidos por el mismo método multiobjetivo. En consecuencia, debido a la imposibilidad de los métodos tradicionales de realizar la optimización simultanea de varios objetivos en la búsqueda de soluciones, el trabajo propone utilizar Algoritmos Evolutivos Multiobjetivos que permitan encontrar soluciones al problema de referencia, optimizando todos los objetivos propuestos, al mismo tiempo. A diferencia de la solución mono-objetivo, la solución multiobjetivo es un conjunto de soluciones Pareto que contiene a todas las soluciones de compromiso, obtenidas al considerar simultáneamente todas las funciones objetivos. En consecuencia, el planificador responsable de la toma de decisiones obtiene un abanico de posibilidades óptimas, en el sentido Pareto, para elegir la solución que mejor se adecue a sus necesidades.

Una importante ventaja de esta metodología es que los tiempos de corridas de estos algoritmos evolutivos son considerablemente más cortos que los requeridos para calcular un conjunto similar de soluciones Pareto, utilizando repetidamente los métodos tradicionales arriba citados. El presente trabajo, propone la optimización de las redes de telecomunicaciones utilizando un Algoritmo Evolutivo Multiobjetivo. En particular, se utilizará el Strength Pareto Evolutionary Algorithm - SPEA2, por su reconocida eficiencia en la búsqueda de soluciones multiobjetivo [6].

Este trabajo está organizado de la siguiente manera: En la sección 1 se formula matemáticamente el problema, exponiendo algunos conceptos relativos a la optimización multiobjetivo, el método utilizado para ubicar las centrales, y el problema de prueba. En la sección 2, se describe el Algoritmo Evolutivo Multiobjetivo propuesto. En la sección 3 se presentan los resultados experimentales obtenidos y su interpretación. Finalmente, se concluye el trabajo en la sección 4.

\section{FORMULACIÓN MATEMÁTICA DEL PROBLEMA}

En esta sección se define algunos conceptos relativos a la optimización Multiobjetivo, se resume el procedimiento realizado para encontrar estas soluciones y se presenta el problema de prueba.

\section{Optimización Multiobjetivo}

El problema de optimización Multiobjetivo tratado en este trabajo, se define de la siguiente forma [7, 8]:

$$
\text { Minimizar } \boldsymbol{y}=\boldsymbol{f}(\boldsymbol{x})=(f 1(\boldsymbol{x}), f 2(\boldsymbol{x}), f 3(\boldsymbol{x}))
$$

Donde:

$x=(x 1, x 2, \ldots, x i, \ldots, x n) ? X ? N^{n}$ representa el vector de decisión;

$y=(y 1, y 2, y 3)=f(x)$ ? Y ? $N^{3}$ representa el vector de objetivos; $n$ : número máximo de centrales;

$m$ : número máximo de cuadrículas en que se divide el área en estudio;

xi: designa la ubicación de una central dentro del área en estudio $(0$ ? xi ? m);

yi : representa la inversión acumulada hasta el año considerado.

Cabe recordar que en un contexto multiobjetivo [8] se dice que un vector objetivo y domina a otro y' sí y solo sí Yi ? yi ', ? ?, y, además, $y j<y j$ ' para por lo menos un $j$.

Una solución $x^{*}$ ? X es Pareto óptima si no existe otra $x$ ? X tal que $y=f(x)$ domine a $y^{\star}=f\left(x^{\star}\right)$. El conjunto de todas las soluciones Pareto óptimas es denominado conjunto Pareto óptimo P (P ? X), y su imagen, Frente Pareto FP (FP ? Y ).

\section{Ubicación Óptima de Centrales y Problema de Prueba}

El problema de la ubicación óptima de centrales, consiste en encontrar el número óptimo de centrales telefónicas, y la mejor ubicación de dichas centrales en un área de estudios (típicamente una ciudad determinada.), de forma a minimizar el costo acumulado de inversión a corto, mediano y largo plazo. El área de la ciudad a ser atendida se divide en $m$ cuadrículas de por ejemplo 10 a $500 \mathrm{~m}$ de lado. A cada una de éstas cuadrículas se le asigna un valor de fila y columna, conformando una matriz. A cada elemento de esta matriz se asocian dos valores: Población, que es la cantidad de habitantes que hay en cada cuadrícula, y Costo del Terreno (por metro cua- 
drado). Los datos de población y terrenos se obtienen a partir de datos oficiales disponibles sobre el área en estudio, que para el presente trabajo, será una ciudad.

De esta forma, se obtiene una matriz $M$ ? $\mathrm{N}^{m \times 4}$ con una fila por cada una de las $m$ cuadrículas válidas y 4 columnas con información por cuadrícula, de:

$1^{\circ}$ columna: fila para su ubicación en el mapa;

$2^{\circ}$ columna: columna para su ubicación en el mapa;

$3^{\circ}$ columna: población actual (dato utilizado para estimar demanda);

$4^{\circ}$ columna: costo del terreno.

Debido a que el plano del área en estudio tiene en general una figura geométrica irregular, muchas cuadrículas caen fuera de los límites de la ciudad o en zonas no habitadas, con ríos, lagos 0 montañas. Por lo tanto, utilizando técnicas de matrices esparzas, a todas las cuadrículas que quedan fuera de la ciudad se les asigna un indicador de cuadrícula no válida (flag) y no se las cuenta entre las $m$ cuadrículas válidas.

El costo de implementación de una central de telecomunicaciones es calculado de la siguiente forma:

$$
\mathrm{Yi}=?_{j ? 1}^{6} \operatorname{cj}(x) \quad \mathrm{i}=1,2,3 \quad \text { (2) }
$$

Donde:

$c_{1}(x)$ : Costo total de planta externa, definidas por el vector de decisión $x$;

$c_{2}(x)$ : Costos de terrenos donde serán instaladas las centrales; $c_{3}(x)$ : Costos de edificios donde serán instaladas las centrales; $c_{4}(x)$ : Costos de ingeniería que conlleva la instalación de las centrales; $c_{5}(x)$ : Costos de equipos de conmutación;

$c_{6}(x)$ : Costos de equipos de transmisión entre las centrales definidas por $x$. Para la evaluación del costo de planta externa $c_{1}(x)$, se calcula las distancias de cada abonado a la central más cercana, conforme se ilustra en el siguiente ejemplo.

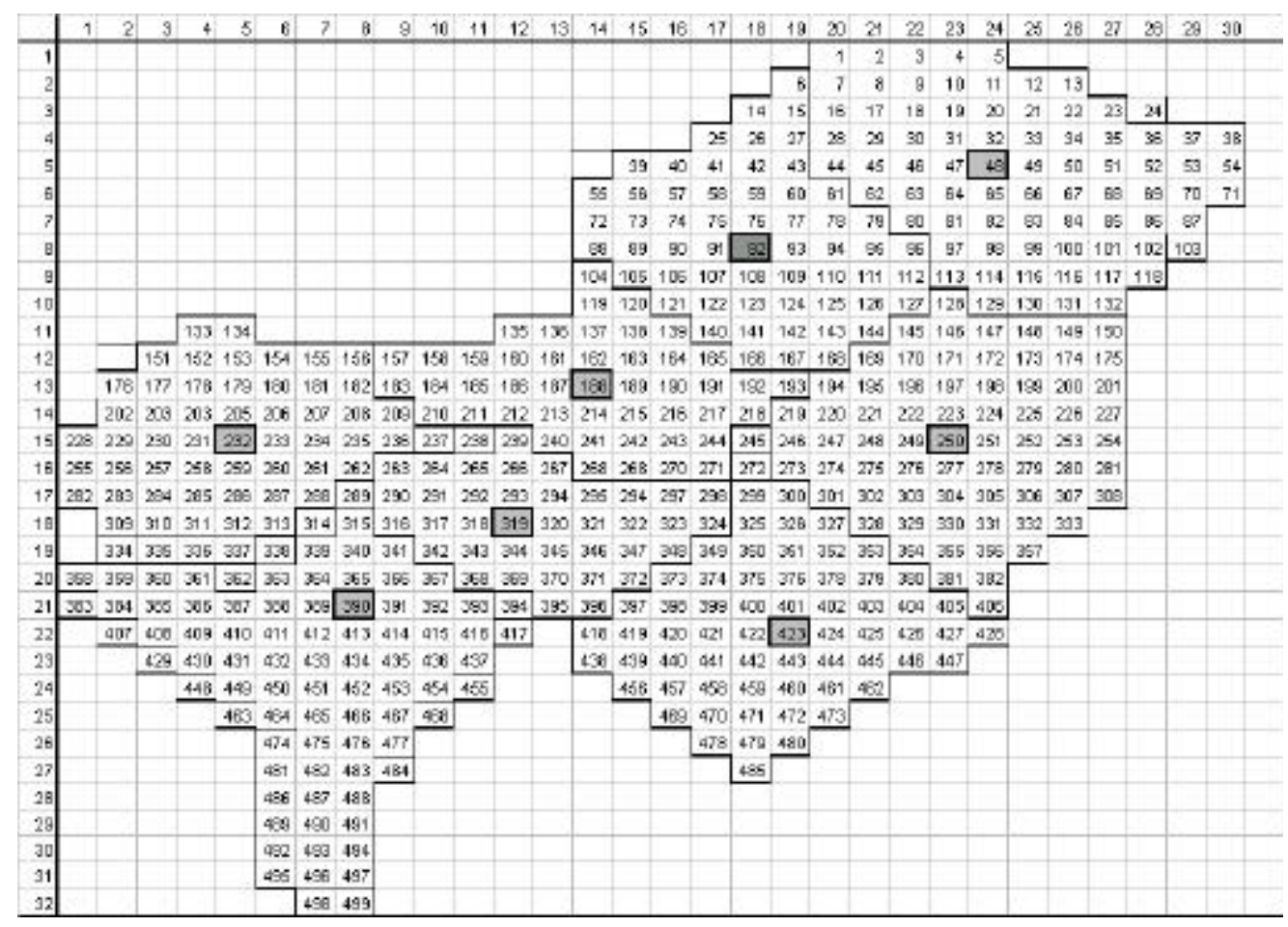

Figura 1. Ejemplo de división en cuadriculas de la ciudad 


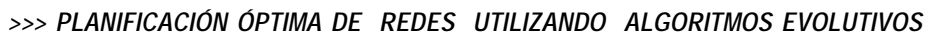

\section{Problema de Prueba}

Como problema de prueba para ejemplificar la presente propuesta se escogió el diseño de la planta externa de una empresa de telefonía, dada la disponibilidad de datos para la misma. El problema, consiste en dado que existen actualmente 8 centrales instaladas en la ciudad, en donde se quiere agregar nuevas centrales de tal forma que se minimicen los costos de ampliación a corto, mediano y largo plazo.

La Figura 1 representa el plano cuadriculado de una ciudad, con los contornos indicando los elementos válidos de la matriz.

Para este ejemplo, existen $m=499$ cuadrículas válidas. El vector de decisión para este ejemplo, al adoptar un número máximo de $n=14$ centrales, será:

$x=(0,0,0,0,0,0,48,92,188,232,250,319,390,423)$

Donde se observa que de las 14 centrales posibles, esta disposición utiliza solo 8 centrales, ubicadas en las posiciones 48, 92 , $188,232,250,319,390$, y 423. Puede notarse, además, que e vector de decisión $\boldsymbol{x}$ tiene sus elementos $x_{i}$ ordenados en forma creciente, lo que facilita detectar soluciones similares donde las centrales se encuentran simplemente permutadas.

Las cuadrículas que formarán parte del área de servicio de una central, son aquellas que tienen el costo mínimo de conexión cuando estan conectadas a esta central. Cada una de estas cuadrículas, denotadas en adelante $x_{t}$, atiende la condición:

1 ? $x_{t}$ ? m. A cada cuadrícula $x_{t}$ van asociados dos valores que representan sus coordenadas $\left(X_{t}, Y_{t}\right)$ en una matriz de 32 filas por 30 columnas. El cálculo del costo de conectar los abonados que están en una cuadrícula xt a una central, se realiza conforme a:

$$
c_{i t}=\text { dt } x \text { p } x\left(\left|X_{i}-X_{t}\right|+\left|Y_{i}-Y_{t}\right|+1\right)
$$

Donde:

$c_{i t:}$ : costo de conectar los abonados pertenecientes a la cuadrícula $x$ a la central $x$;

$D_{t}$ : Cantidad de abonados de la cuadrícula $x_{t}$;

p: Costo de planta externa por unidad de longitud, por cada abonado;

$\left(X_{i}, Y_{j}\right)$ : Coordenadas de la central $X_{i}$

$\left(X_{t}, Y_{t}\right)$ : Coordenadas de la cuadrícula $X_{t}$.

El área de servicio de cada central contiene aquellas cuadrículas con menores distancias a dicha central, de forma a minimizar el costo de planta externa $c_{1}(x)$. Por lo tanto, el costo total de planta externa, para el ejemplo considerado, se calcula conforme:

$$
\mathrm{C} 1(\mathrm{x})=?^{n}{ }_{i ? 1}^{n} \quad ?^{m}{ }_{i ? 1}^{m} \quad \mathrm{c}_{\mathrm{it}}-\mathrm{h}_{\mathrm{it}}
$$

hit $=1$ Si el sitio $x_{t}$, puede estar conectado a una sola central $x_{i} ; 0$ caso contrario.

Se asume que cada sitio xt puede estar conectado a una sola central xi, por lo tanto:

$$
?_{i ? 1}^{n} \quad \mathrm{~h}_{\mathrm{it}}=\mathbf{1} \quad \mathrm{x}_{\mathrm{t}}=1,2 \ldots \ldots . \mathrm{m} .
$$

El costo del terreno es el producto del costo por $\mathrm{m}^{2} \mathrm{y}$ el área del edificio de la central, conforme:

$$
\mathrm{C} 2(\mathbf{x})=?_{i ? 1}^{n} \quad \mathbf{q}_{\mathbf{i}} \mathbf{x g}_{\mathbf{i}} \mathbf{x} \mathbf{w}_{\mathbf{i}}
$$

q: área en $\mathrm{m}^{2}$ a ser ocupada por la central $x i$

$g_{i:}$ costo del terreno por $m 2$ en la cuadrícula $x_{i}$. $\mathrm{w}_{\mathrm{i}}: 1$ si la central esta en el sitio $x_{i} ; 0$ caso contrario.

$$
\begin{gathered}
c_{3}(x)=?_{i ? 1}^{n} \quad c_{e} x w_{i} \\
c_{4}(x)=?_{i ? 1}^{n} \quad c_{\text {ing }} x w_{i} \\
c_{5}(x)=?_{t ? 1}^{m} \quad c_{e q} \quad x d t \\
c_{6}(x)=\left(\begin{array}{ll}
c_{\text {tr }} & x k x(k-1)) / 2 \\
K & w_{i}^{n}
\end{array}\right.
\end{gathered}
$$

Donde:

$c_{e}$ : Costo de construcción del edificio de la central;

$c_{i n g}$ : Costo de la ingeniería de planificación de centrales;

$c_{e q}$ : Costo de los equipamientos de la central, por abonado;

$c_{t r}$ : Costo de los equipamos. de transmisión;

$k$ : Cantidad de centrales $(k$ ? $n)$;

$d_{t}$ : Demanda telefónica de la cuadrícula $x_{t}$

En consecuencia, el problema principal a ser resuelto consiste en encontrar la cantidad de centrales y la ubicación óptima de estas centrales en el área de estudio, de la cual se conocen todos los datos relativos a la matriz $M$ arriba definida. Si existen $m$ sitios posibles, existen claramente $2 m$ alternativas de ubicación de centrales. Aún, si se restringe la atención para ubicar $n$ centrales en $m$ sitios, el número de alternativas de ubicación de centrales es todavía:

$$
C_{n}^{m}=\mathbf{m} ! /(\mathbf{m}-\mathbf{n}) ! \mathbf{n} ! \quad \text { (8) }
$$


En el ejemplo de la Figura 1, para 499 cuadrículas válidas y 14 centrales, existen unas $5,66 \times 10^{26}$ alternativas de ubicación de centrales. El problema propuesto en el presente trabajo, permite encontrar soluciones Pareto que minimicen los costos acumulados a corto, mediano y largo plazo, de un conjunto de alternativas de ubicación de centrales, considerando los diferentes valores posibles del número $k$ de centrales $(k ? n)$. El espacio de búsqueda del problema propuesto, es entonces:

$$
?_{i ? 1}^{n} \quad\left(C_{i}^{m}\right)
$$

En otras palabras, el método a ser utilizado en el presente trabajo debe posibilitar la obtención de un conjunto de soluciones Pareto óptimas, estableciendo la cantidad y la ubicación óptima de estas centrales.

\section{ALGORITMO EVOLUTIVO PROPUESTO}

El algoritmo evolutivo propuesto es el SPEA2 cuya eficiencia en la búsqueda de soluciones se caracteriza por la obtención de soluciones Pareto óptimas y la diversidad de las mismas sobre el Frente Pareto. Este algoritmo utiliza una estrategia de asignación de fitness que incorpora información de densidad a fin de evitar la pérdida de posibles soluciones óptimas [8]. El operador de truncamiento elimina aquellos individuos que están muy pegados unos a otros de forma a no perder puntos valiosos de la frontera y asegurar de esta forma que las soluciones encontradas en el frente Pareto, sean regularmente distribuidas. El proceso de encontrar los individuos no dominados en el archivo y la población, está basado en el concepto de dominancia Pareto. Cada vez que un individuo no dominado es encontrado, el mismo es comparado con los no dominados ya existentes en el archivo, y si el mismo es una solución, el individuo hallado es insertado en el archivo. Para esclarecer el procedimiento de aplicación del SPEA2 en la planificación de centrales, a continuación se presenta un esquema de utilización del referido algoritmo.

\section{Representación de Soluciones y Población Inicial}

Para la aplicación de los Algoritmos Evolutivos Multiobjetivos propuestos en el problema de prueba, cada individuo $x=(x 1, x 2, \ldots$, $x i, \ldots, x n)$ fue codificado usando un arreglo de números enteros $x i$, tal que 0 ? xi ? $m(m=499)$.

En la Figura 1, donde se representa el plano cuadriculado de la ciudad, se puede apreciar los 499 valores no nulos de la matriz utilizada para los cálculos de costos de cada vector de decisión. La población inicial, cuyo tamaño se denotará como "nind" (número de individuos), es generada por un algoritmo heurístico de inicialización, en donde "nmax"indica el número máximo de centrales para cada vector de decisión. Este algoritmo genera una población inicial en forma inteligente de manera a obtener individuos que se aproximen razonablemente al conjunto de soluciones Pareto óptimas buscadas, minimizando de esta forma los tiempos de corridas. Para cada individuo de la población, se realiza un sorteo para saber cuantas centrales tendrá esa solución, y se ubican las centrales de tal forma a que las mismas estén ubicadas en los centros de demandas a fin de minimizar los costos de conexión de los abonados a su central correspondiente. El algoritmo heurístico de inicio de la población se describe en el Pseudocódigo 1: Algoritmo Heurístico de generación de la población inicial.

\section{Evaluación de Soluciones y Función Fitness}

En la evaluación de la función fitness, se utilizaron los conceptos de dominancia Pareto definidos en la sección 1.1 en un contexto de minimización de funciones objetivos. De esta forma, cada vector de decisión es comparado con otro a través de las funciones objetivos de dichos vectores, de tal forma a determinar si un individuo $i$ domina a otro individuo $j$. La función fitness $(x)$ fue implementada conforme a lo especificado por el SPEA2 de Zitzler [1]. Los valores de fitness calculados mediante este algoritmo, son utilizados en la selección de los individuos que pasarán a formar parte del archivo que contiene a los mejores individuos de la población. El referido algoritmo asigna a los individuos no dominados un fitness menor a 1 , en cuanto que a los individuos dominados se les asigna un fitness mayor 0 igual a 1, con lo que todos los individuos tienen diferentes valores de fitness.

Algoritmo Heurístico de Inicialización de la Población Inicial. Leer parámetros: nind, nmax

Ordenar matriz de población de acuerdo al número de habitantes Para in $=1$ hasta nind

Generar un número aleatorio $N$ entre 6 y $n$ max

Dividir la población total en $N$ partes:

Parte $=$ población.total $/ N$

Para $i=1$ hasta $N$

Elegir punto $x i$ aleatoriamente entre las 5 ubicaciones más pobladas

Hallar distancia euclidiana de $x i$ a todas las ubicaciones de la matriz de población

Ordenar las distancias obtenidas de menor a mayor

poblacion $=0$

Mientras poblacion es menor 0 igual a parte

Sumar a poblacion la población de las ubicaciones más próximas axi

Fin Mientras

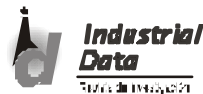

diciembre 2003 
Eliminar de la matriz de población las ubicaciones que se agregaron a población

Hallar el centro geométrico Pi de todas las ubicaciones que se agregaron a población

Hacer $x i=P i$

Fin Para

Si $N<n \max$

$x i=0$ para todo i que no contiene una central (esto es, $N+1=i$ ? nmax) Fin Si

Fin Para

Eliminar centrales repetidas de cada individuo de la población inicial y ordenar centrales en orden creciente fitness mayor 0 igual a 1 , con lo que todos los individuos tienen diferentes valores de fitness.

\section{Selección}

Se denomina como selección del ambiente a la acción de completar con los mejores individuos de cada generación, una población externa denominada archivo. El tamaño del archivo es fijo y no varía durante las corridas del algoritmo. Inicialmente, todos los individuos no dominados, cuyos fitness son menores que uno, son copiados al archivo de la siguiente generación:

$$
\mathrm{P}_{\mathrm{t}+1}=\left[\mathrm{i} / \mathrm{i} ? P_{t} * P_{t}^{\wedge} F(i)<1\right]
$$

Si la cantidad de individuos no dominados es igual al tamaño establecido para dicho archivo $(|\mathrm{Pt}+1|=\mathrm{N})$, el paso de selección del ambiente está completo. Caso contrario, existen dos posibilidades:

1. La cantidad de individuos no dominados es menor que el tamaño establecido para el archivo $(|\mathrm{Pt}+1|<\mathrm{N})$,

2. La cantidad de no dominados es mayor que el tamaño fijado para el archivo $(|\mathrm{Pt}+1|>\mathrm{N})$.

En el primer caso, se completa el archivo con los mejores ( $N$ $|\mathrm{Pt}+1|)$ individuos dominados en el archivo y la población de la generación anterior $t$. Esto es implementado ordenando el multiconjunto t $P_{t}+P_{t}$ de acuerdo a los valores de fitness y copiando a $P_{t+1}$ los primeros ( $\mathrm{N}-|\mathrm{Pt}+1|$ ) individuos $i$ con fitness $F(i)>1$. En el segundo caso, cuando el tamaño del conjunto de no dominados es mayor a $N$, un operador de truncamiento remueve iterativamente los individuos de $P_{t+1}$ hasta que el conjunto de no dominados sea igual al tamaño establecido para el archivo $\left|P_{t+1}\right|=\mathrm{N}$.

Este operador de truncamiento garantiza que puntos valiosos de la frontera no sean perdidos, y lo realiza de la siguiente forma: el individuo que tiene la menor distancia euclidiana a otro individuo es desechado en cada iteración.

En caso de igualdad con otros individuos, se desempata considerando la segunda menor distancia del individuo a ser removido, y así sucesivamente.

Pseudocódigo del Algoritmo Evolutivo Multiobjetivo

\section{Propuesto SPEA 2}

En las corridas realizadas del algoritmo SPEA 2 se utilizaron los siguientes parámetros:

- Tamaño de la población (nind) $=100$.

- Número máximo de centrales (nmax) = 14 a 20.

- Tamaño del archivo de no dominados (nptrue) $=100$.

- Número máximo de generaciones (ngen) = 1000 a 3000 .

- Probabilidad de cruzamiento $(\mathrm{pc})=0,7$ a 0,9.

- Probabilidad de mutación (pm) =0,1 a 0,3.

A continuación, se presenta el Pseudocódigo del algoritmo Multiobjetivo utilizado

\section{Programa Principal SPEA 2}

Leer los parámetros del SPEA 2: nind, nmax, ngen, pm, pc, nptrue

Generar una población usando el algoritmo heurístico

(Pseudocódigo 1)

Generar un archivo vacío (conjunto externo)

Para gen $=1$ hasta ngen

Eliminar centrales repetidas del individuo

Evaluar funciones objetivo de cada individuo de la población Asignar fitness a cada individuo de la población y del archivo Calcular todos los individuos no dominados de la población y el archivo

Actualizar el archivo con los individuos no dominados

Si el tamaño del archivo es mayor que nptrue Reducir el tamaño del archivo con el operador de truncamiento

Caso contrario

Si el tamaño del archivo es menor que nptrue Copiar los mejores individuos dominados del archivo y la población con fitness ? 1 al archivo de la nueva generación hasta que el tamaño del archivo sea igual a nptrue

Fin Si 
Cuadro 1. Soluciones encontradas de ampliación de centrales del problema de prueba

\begin{tabular}{|c|c|c|c|c|c|c|c|c|c|c|c|c|c|c|c|c|c|}
\hline & & & . & & & $\mathbf{R}$ & co & 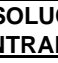 & ION & & & & & & & STOS EN U & \\
\hline Solució & & & & & & & ecto & decis & ión & & & & & & Año 2002 & Año 2004 & Año 2007 \\
\hline 1 & 0 & 0 & 0 & 0 & 0 & 48 & 92 & 188 & 232 & 250 & 319 & 373 & 390 & 423 & 299870 & 51614ó5 & 12708016 \\
\hline 2 & 0 & 0 & 0 & 0 & 48 & 92 & 147 & 188 & 232 & 250 & 319 & 373 & 390 & 423 & 723880 & 5161349 & 12732746 \\
\hline 3 & 0 & 0 & 0 & 48 & 92 & 147 & 188 & 232 & 250 & 319 & 337 & 373 & 390 & 423 & 1331780 & 5162809 & 12721563 \\
\hline 4 & 0 & 0 & 0 & 48 & 92 & 147 & 188 & 232 & 250 & 319 & 338 & 373 & 390 & 423 & 1341380 & 5162695 & 12721195 \\
\hline 5 & 0 & 0 & 0 & 48 & 92 & 147 & 188 & 232 & 250 & 263 & 319 & 373 & 390 & 423 & 1346220 & 5161426 & 12717966 \\
\hline 6 & 0 & 0 & 48 & 92 & 147 & 184 & 188 & 232 & 250 & 319 & 337 & 373 & 390 & 423 & 2053060 & 5164523 & 12710869 \\
\hline 7 & 0 & 0 & 48 & 92 & 147 & 188 & 232 & 250 & 263 & 319 & 337 & 373 & 390 & 423 & 2054120 & 5162886 & 12706783 \\
\hline 8 & 0 & 0 & 48 & 92 & 147 & 188 & 232 & 250 & 263 & 312 & 319 & 373 & 390 & 423 & 2102530 & 5162904 & 12706739 \\
\hline 9 & 0 & 48 & 92 & 147 & 184 & 188 & 232 & 250 & 319 & 337 & 355 & 373 & 390 & 423 & 2906550 & 5166569 & 12701059 \\
\hline 10 & 0 & 48 & 92 & 147 & 188 & 232 & 250 & 263 & 319 & 337 & 355 & 373 & 390 & 423 & 2907610 & 5164933 & 12696973 \\
\hline 11 & 0 & 48 & 92 & 147 & 188 & 232 & 250 & 263 & 319 & 330 & 337 & 373 & 390 & 423 & 2926340 & 5164740 & 12696404 \\
\hline 12 & 0 & 48 & 92 & 147 & 188 & 232 & 250 & 263 & 272 & 319 & 337 & 372 & 390 & 423 & 3065780 & 5164478 & 12696273 \\
\hline 13 & 48 & 92 & 147 & 188 & 232 & 250 & 263 & 319 & 324 & 337 & 355 & 390 & 397 & 423 & 3985070 & 5168055 & 12690033 \\
\hline 14 & 48 & 92 & 147 & 188 & 232 & 250 & 263 & 271 & 319 & 337 & 355 & 372 & 390 & 423 & 4017810 & 5167303 & 12688326 \\
\hline 15 & 48 & 92 & 147 & 188 & 232 & 250 & 263 & 272 & 319 & 337 & 355 & 372 & 390 & 423 & 4028190 & 5166664 & 12686821 \\
\hline 16 & 48 & 92 & 147 & 188 & 232 & 250 & 263 & 272 & 319 & 330 & 337 & 372 & 390 & 423 & 4055950 & 5166620 & 12686611 \\
\hline
\end{tabular}

Si gen es menor que ngen

Realizar torneo binario para seleccionar los

individuos del archivo que formarán parte

del conjunto de emparejamientos

Realizar cruzamiento y mutación del conjunto de emparejamientos

Actualizar la población del resultado del

conjunto de emparejamientos

Fin Si

Fin Para

Incrementar contador de generaciones (gen=gen+1)

Salvar el archivo (conjunto de no dominados)

Pseudocódigo2: Algoritmo SPEA2 implementado.

\section{RESULTADOSEXPERIMENTALES}

Las soluciones obtenidas para el problema de prueba son presentadas en el Cuadro 1, las mismas fueron obtenidas mediante sucesivas corridas del algotritmo SPA2, luego de haber descartado otros algoritmos evolutivos que no lograron el nivel de desempeño obtenido con el SPEA2.
En el Cuadro 1 se puede apreciar que la mejor solución para el año base 2002 es la número 1, que utiliza 9 centrales.

Para el año 2004, la mejor solución es la número 2 que requiere de 10 centrales, mientras que para el año 2007, la cantidad óptima de centrales es de 14 (solución número 16). Claramente, los tres objetivos conflictúan entre sí por lo que el planificador deberá decidir cual es la mejor relación de compromiso entre su inversión a corto plazo y el costo que podrá llegar a tener la red a mediano y largo plazo. Es interesante enfatizar que al utilizar un algoritmo evolutivo multiobjetivo, el planificador no sólo encuentra las mejores soluciones para cada objetivo, sino toda la gama de soluciones de compromiso Pareto óptimas entre estos objetivos, por lo que se facilita la toma de decisión consciente.

Cabe mencionar que la solución efectivamente implementada para la ciudad no es una solución Pareto óptima, y de hecho conlleva un costo mucho mayor que cualquiera de las soluciones calculadas con la metodología propuesta, sin importar cual de las 3 funciones objetivos se considere.

En este procedimiento descrito, el planificador puede elegir una de las soluciones encontradas en el Cuadro 1 y dar énfasis a un solo objetivo. Por ejemplo, se podrá elegir la solución número 16 


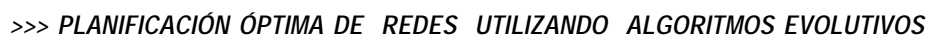

en donde el costo de inversión inicial para el año base 2002 es más elevado, pero tendrá importantes ahorros, cuando se necesite realizar ampliaciones en los años 2004 y 2007.

Dado que en la metodología propuesta existen varias soluciones no dominadas entre sí, y a fin de simplificar la tarea del planificador, se presenta en la Figura 2 una sugerencia pragmática para elegir una de entre todas las soluciones Pareto óptima. La idea es traer a valor presente las inversiones a mediano y largo plazo de forma a tener un único objetivo que permita comparar todas las alternativas de solución encontradas por el planificador, en el tradicional contexto mono-objetivo. En la Figura 2 puede notarse que en la simplificación propuesta, el número óptimo de centrales es 9.

\section{CONCLUSIONES}

La utilización de Algoritmos Evolutivos Multiobjetivos en la resolución de problemas de ubicación de centrales de telecomunicaciones, presenta un enfoque inédito en la planificación de redes de telefonía básica. Esta metodología proporciona una herramienta computacional que permite obtener un conjunto de soluciones Pareto óptimas, considerando todos los aspectos que se quieran optimizar de manera simultánea. Los métodos heurísticos tradicionales proporcionan simplemente soluciones puntuales [6], apelando a procesos iterativos para contemplar todos los aspectos de la red que se quiere diseñar, con la consecuente demora en el diseño.

Con el presente trabajo, se nota que la utilización de algoritmos evolutivos Multiobjetivos como el SPEA2, proporciona al

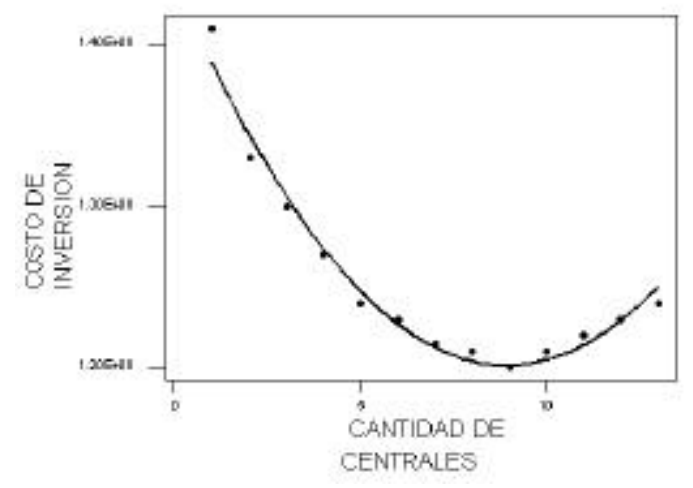

Figura 2. Valor presente del proyecto vs. Cantidad de centrales planificador de redes un conjunto de soluciones Pareto óptimas para la correcta ubicación de las centrales, de forma a minimizar los costos iniciales de inversión y las inversiones de expansión a mediano y a largo plazo. Conforme con los resultados obtenidos en este trabajo, se puede aseverar que las soluciones distribuidas sobre el frente Pareto son dominantes con respecto a las soluciones efectivamente implementadas por empresas del área que se limitaron a utilizar herramientas tradicionales de cómputo en sus estudios de planificación. De hecho, en las pruebas realizadas, las soluciones obtenidas con el SPEA2 superaron claramente a las obtenidas por otros métodos tradicionales.

En definitiva, se puede afirmar que el empleo de algoritmos evolutivos Multiobjetivos para la planificación, dimensionamiento y optimización de redes de telecomunicaciones, ofrece una perspectiva más amplia y eficiente que permite a los planificadores decidir entre un conjunto de soluciones óptimas, manejando los diversos aspectos de la red que se consideren necesarios para minimizar los costos en juego.

Cabe destacar que la metodología adoptada para resolver el problema de ubicación de centrales es fácilmente adaptable a otros problemas similares. Por ejemplo, dado una cantidad de centrales existentes en un área, se puede calcular donde agregar nuevas centrales para satisfacer la demanda a 5 y 10 años de plazo.

Sobre la base de los resultados obtenidos, se puede utilizar la metodología propuesta para mejorar: la planificación de redes de telecomunicaciones, ubicación de estaciones bases para telefonía celular, o en general, ubicar de manera óptima centros de atendimiento de diversos servicios, como cadenas de comidas rápidas, supermercados, etc. La simplicidad de la metodología propuesta, para un problema tan complejo, alienta a mirar con optimismo la realización de futuros trabajos en el área, así como nuevas aplicaciones.

\section{BIBLIOGRAFÍA}

1. Arroyo, J. y Armentano, V. (2001). Um Algoritmo Genético para Problemas de Otimizaçao Combinatoria Multiobjetivo, XXXIII Simpósio Brasileiro de Pesquisa Operacional. Campos do Jordao - SP. Noviembre, 2001.

2. Baran, B. and Duarte, S. (2002). Multiobjective Network Design Optimization using Parallel Evolutionary Algorithms. Centro Nacional de Computación, Universidad Nacional de Asunción. San Lorenzo, Paraguay. Agosto, 2002. 
3. Boorstyn, R. R. and Frank, H. (1997). Large-Scale Network Topological Optimization, IEEE Transactions on Communications, Vol. COM-25, No. 1, Enero 1977

4. Cooper, L. and Steinberg, D. (1974). Methods and Applications of Linear Programming. Saunders, Philadelphia, 1974.

5. Glover F., Laguna M., Taillard E., and Werra D. (1993). De:Tabu Search, special issues of Annals of Operations Research, Vol. 41, No. 1-4. J. C. Baltzer Science Publishers, Basel, Switzerland, 1993.
6. PLANITU. (1984). UIT: Programas de Planificación de Redes, Vol. 1, Documentación Básica, Edición Preliminar, Junio 1984.

7. Robertazzi, T. G. (1998). Planning Telecommunication Networks, IEEE Press, IEEE Communications Society.

8. Zitzler E., Laumanns M., and Thiele L. (2001). SPEA2: Improving The Strength Pareto Evolutionary Algorithms, Technical Report 103, Computer Engineering and Networks Laboratory, Swiss Federal Institute of Technology. Zurich, Switzerland, Mayo 2001. 